\title{
Interstellar extinction by composite grains
}

\author{
D. B. Vaidya ${ }^{1,2}$, R. Gupta ${ }^{2}$, J. S. Dobbie ${ }^{3}$, and P. Chylek ${ }^{3}$ \\ 1 Gujarat College, Ahmedabad 380009, India \\ e-mail: vaidya@iucaa.ernet.in \\ 2 IUCAA, Post Bag 4, Ganeshkhind, Pune 411007, India \\ 3 Dept. of Physics, Dalhousie University, Halifax, Nova Scotia, B3H 3J5 Canada
}

Received 11 December 2000 / Accepted 14 June 2001

\begin{abstract}
Recent studies indicate that interstellar grains are composites of very small individual particles of silicates and carbon glued together into an aggregate. Using the discrete dipole approximation (DDA), we calculate the extinction, scattering and absorption efficiencies for composite grains assumed to be made of a host silicate sphere with embedded graphite inclusions. In particular, we study the extinction as a function of inclusion size and volume fraction. Using the extinction efficiencies of these composite grains, we evaluate the interstellar extinction curve in the wavelength region of $0.55 \mu \mathrm{m}-0.20 \mu \mathrm{m}$.
\end{abstract}

Key words. ISM: dust - extinction

\section{Introduction}

Recent studies of interplanetary, cometary and interstellar dust indicate that cosmic dust grains are inhomogeneous and are composites of many small particles. Hence there is a need to formulate models of electromagnetic scattering by composite dust grains. Mathis \& Whiffen (1989), Mathis (1996) and Voshchinnikov \& Mathis (1999) have used effective medium theory (EMT) to calculate the optical constants for composite grains and then used Mie theory to study the scattering properties of the approximated dust particles. We use the discrete dipole approximation (DDA)(Draine 1988) to study the scattering properties of the composite grains. For a discussion and comparison of DDA and EMT methods see for example Perrin \& Lamy (1990) and Wolff et al. (1994, 1998). Mathis (1996) has noted the uncertainty in the use of EMT for treating the composite grains and has suggested that detailed calculations such as the DDA would be necessary for the treatment of voids or silicate/carbon particles for some wavelengths. In the previous work we have used the DDA to study the scattering properties of porous grains (Vaidya \& Gupta 1997, 1999, hereafter called as Papers I and II). We have also used the DDA to study the scattering properties of a composite particle made up of a host water sphere with carbon inclusions (Dobbie \& Chylek 1998 and Chylek et al. 1999). In the present study, we are using a modified version of the DDA code (Dobbie 1999; Dobbie \& Chylek 1998), and we will calculate the extinction efficiencies of the composite grains, assumed to be made of the

Send offprint requests to: R. Gupta,

e-mail: rag@iucaa.ernet.in host silicate sphere with embedded inclusions of graphite. In particular, we study the extinction as a function of inclusion size and volume fraction.

Mathis (1996) has suggested that carbon and silicate occur separately in small particles in the interstellar medium and are agglomerated into large grains, in which $25-50 \%$ vacuum arises from the coagulation. In addition to composite grains, small graphite grains are required in order to produce the observed " $2175 \AA$ " feature. In this present study, we use the results of the composite grain model to evaluate the interstellar extinction curve in the wavelength region of $0.20 \mu \mathrm{m}-0.55 \mu \mathrm{m}$ and in particular, we are interested in studying the wavelength region of peak extinction, i.e. $0.21 \mu \mathrm{m}-0.23 \mu \mathrm{m}$.

We present the composite dust model along with the DDA validity criteria in Sect. 2 . Section 3 gives the main results, including the interstellar extinction curves. In Sect. 4 we provide conclusions on the present study.

\section{Discrete dipole approximation and the composite grain model}

Original formulations of the DDA method sought to analytically solve the coupled dipole equations (Defoe 1965; Purcell \& Pennypacker 1973; Shapiro 1975). Numerical formulation of this problem, however, allowed for a large increase in the number of dipoles that could be used to approximate particles, and so larger and more complex particles could be treated (Yung 1978; Draine 1988).

The DDA replaces a solid particle by an array of $N$ point dipoles. When a grain is exposed to an electromagnetic wave, each dipole responds to the radiation field of 

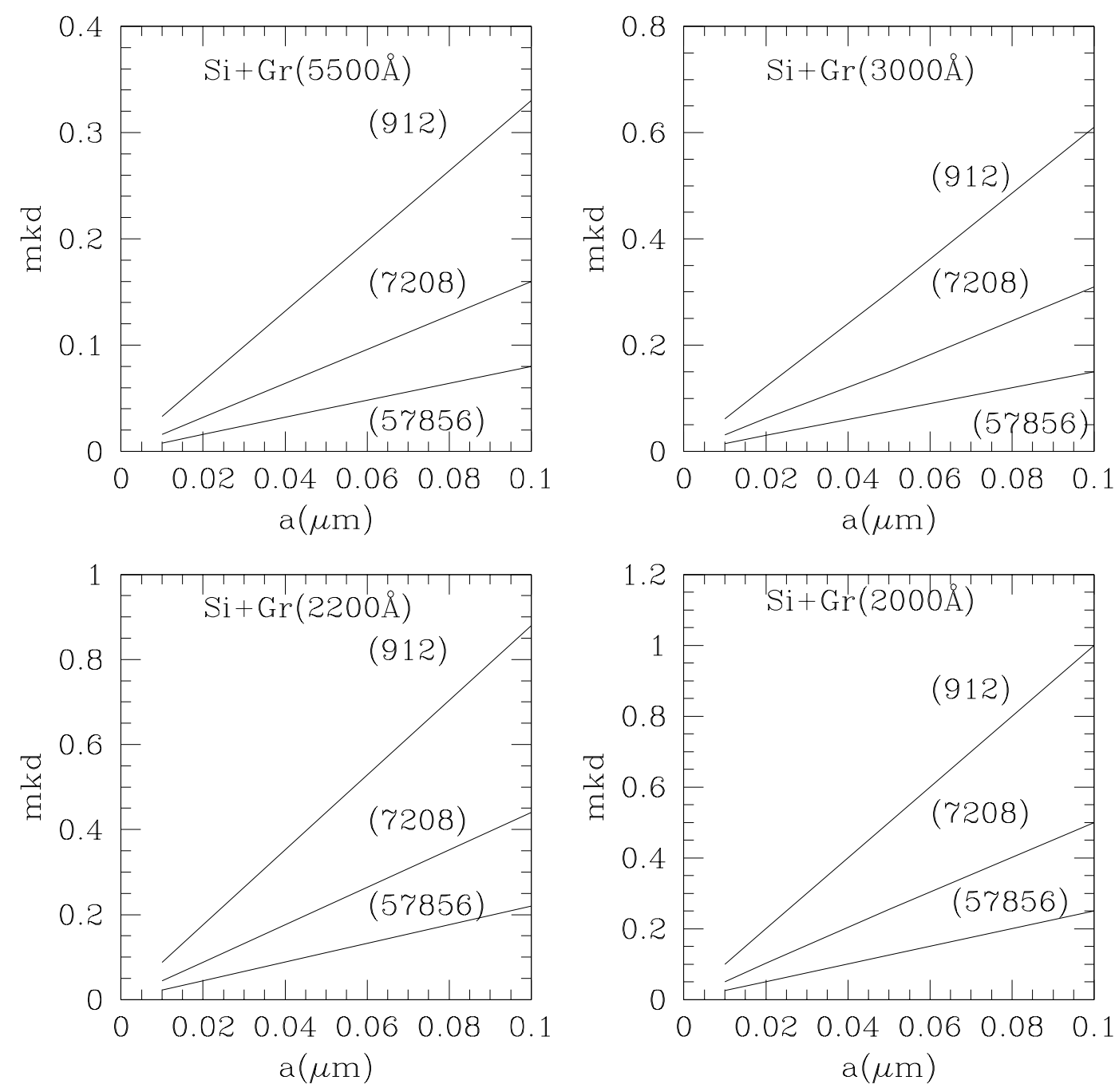

Fig. 1. Lattice dispersion relation $m k d$ as a function of grain size at four wavelengths.

the incident wave as well as to the fields due to all of the other dipoles. There are two validity criteria for DDA (Draine \& Flatau 1994; Wolff et al. 1994): (i) $|m k d|<1$ where $m$ is the complex refractive index, $k$ is the wave number $k=\pi / \lambda$ and $d$ is the distance between the dipoles; and (ii) $d$ should be small enough ( $N$ should be sufficiently large) to describe the shape of the particle satisfactorily.

In the present study we use DDA to calculate the extinction efficiency for a composite grain made up of a host silicate sphere with graphite inclusions. A computer code developed by Dobbie (1999) first carves out an outer sphere from a lattice of dipole sites. Sites outside the sphere are set to be vacuum and sites inside are assigned to be the host material. Second, once the host particle is formed, the code locates centers for internal spheres using a random number generator. If the internal sphere can be formed without extending beyond the host and is not overlapping another inclusion, then the inclusion is formed by assigning the material to have a refractive index of the inclusion type. The code continues locating places for inclusions until the specified volume fraction of the inclusions is reached or exceeded. The inclusions are of a single ra- dius and their centers are chosen randomly. The code then outputs a three-dimensional matrix specifying the material type at each dipole site which is read in by the DDA. In the present case the sites are either silicate, graphite or vacuum. These geometries are then input into the DDA code and the optical properties are computed. For more details on the computer code and the corresponding modification to the DDA code see Dobbie (1999). We have studied composite grain models with a host silicate sphere containing $N=57856,7208$ and 912 dipoles, each carved out from $48 * 48 * 48,24 * 24 * 24$ and $12 * 12 * 12$ dipole sites, respectively. The volume fractions of the graphite inclusions used are $10 \%, 20 \%$ and $30 \%$. The inclusion size is labeled by the number of dipoles " $n$ " across the diameter of an inclusion.

An illustration of the composite grain model is given elsewhere (Vaidya et al. 2000).

Table 1 shows the maximum radius of the host sphere $a$ that satisfies the validity criteria for DDA (i.e. $|m k d|<1$ ) at several wavelengths for the composite models, viz. $N=912,7208,57856$. The complex refractive indices 


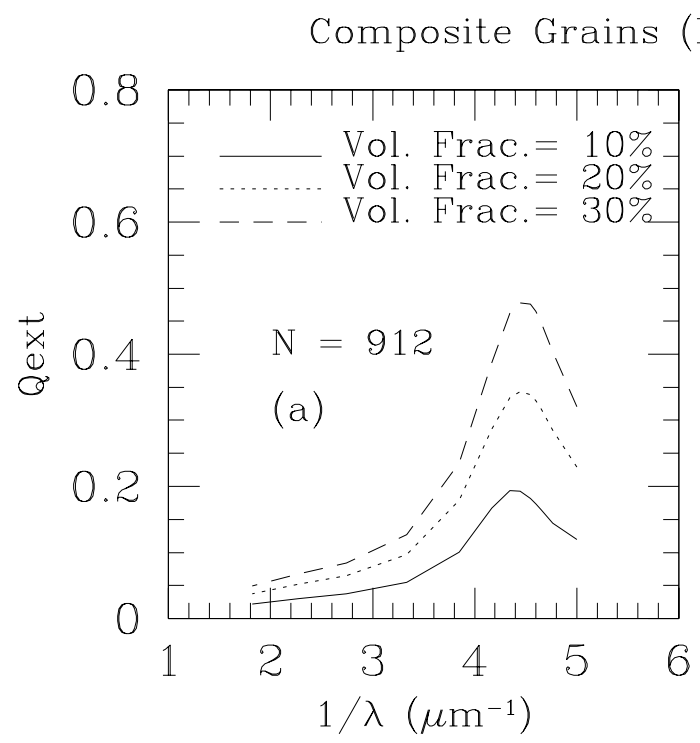

(Host Silicate, Incl. Graphite)
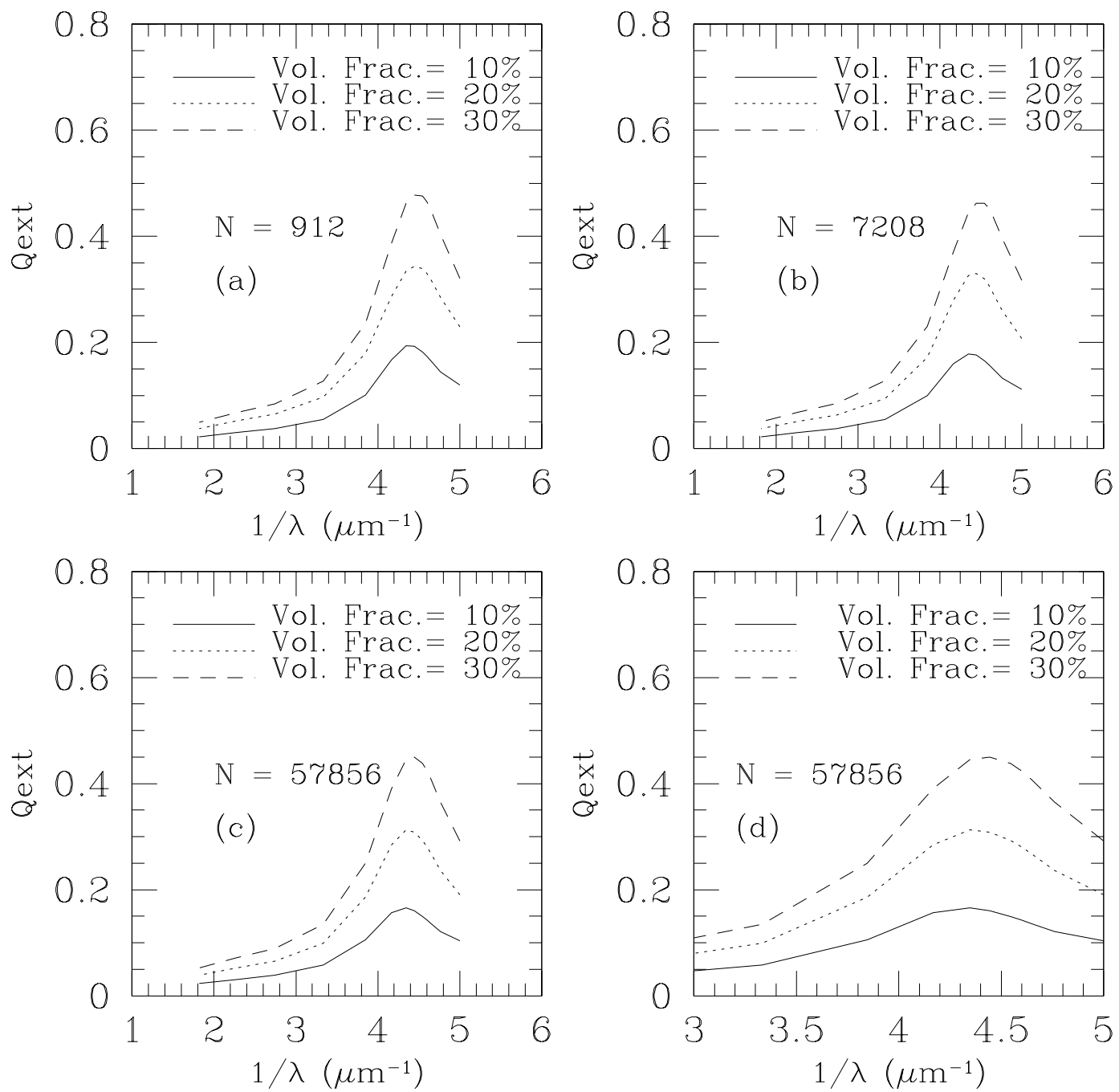

Fig. 2. Extinction efficiencies for the composite grains with host spheres containing 912, 7208 and 57856 dipoles are shown in a), b) and c) respectively. The shift in the peak is highlighted in $\mathbf{d}$ ).

Table 1. Validity criteria: This table shows the maximum radius $(a)$ of the host sphere that satisfies the validity criteria for DDA (viz. $|m k d|<1$ ) at several wavelengths for $N=912$, 7208 and 57856 .

\begin{tabular}{lccc}
\hline \hline Wavelength & $N=912$ & $N=7208$ & $N=57856$ \\
\hline$(\mu \mathrm{m})$ & $\mathrm{a}(\mu)$ & $\mathrm{a}(\mu)$ & $\mathrm{a}(\mu)$ \\
\hline 0.5500 & 0.330 & 0.65 & 1.30 \\
0.4400 & 0.250 & 0.50 & 1.00 \\
0.3000 & 0.170 & 0.33 & 0.66 \\
0.2600 & 0.140 & 0.28 & 0.56 \\
0.2200 & 0.120 & 0.25 & 0.50 \\
0.2175 & 0.110 & 0.22 & 0.44 \\
0.2000 & 0.100 & 0.20 & 0.40 \\
\hline
\end{tabular}

for silicates and graphite are obtained from Draine (1985, 1987).

It is seen from Table 1 that the composite grain model with $N=912$ is valid for the grain sizes upto $0.1 \mu$ at the wavelength $0.20 \mu \mathrm{m}$ whereas models with $N=7208$ and $N=57856$ are valid up to grain sizes $0.20 \mu$ and $0.40 \mu$ respectively, at the same wavelength. At longer wavelengths (i.e. in the optical), the DDA is valid for larger grain sizes for these models. Figure 1 shows the plots of the lattice dispersion relation, $m k d$, as a function of grain size, $a$, at four wavelengths viz. $0.55,0.30,0.22$ and $0.20 \mu \mathrm{m}$.

\section{Results and discussion}

Using the DDA code (Dobbie \& Chylek 1998), we have calculated the extinction efficiencies for the composite grains in the wavelength region of $0.55 \mu \mathrm{m}-0.20 \mu \mathrm{m}$. Figures $2 \mathrm{a}, \mathrm{b}$ and $\mathrm{c}$ show the extinction $\left(Q_{\text {ext }}\right)$ efficiencies for the composite grains with the host silicate sphere containing 912, 7208 and 57856 dipoles, respectively. Within each graph three volume fractions are listed, viz. $10 \%$, $20 \%$ and $30 \%$, of graphite inclusions for a constant inclusion size $n=6$. The radius of the host composite grain is set to $0.01 \mu$ for all of the cases. 


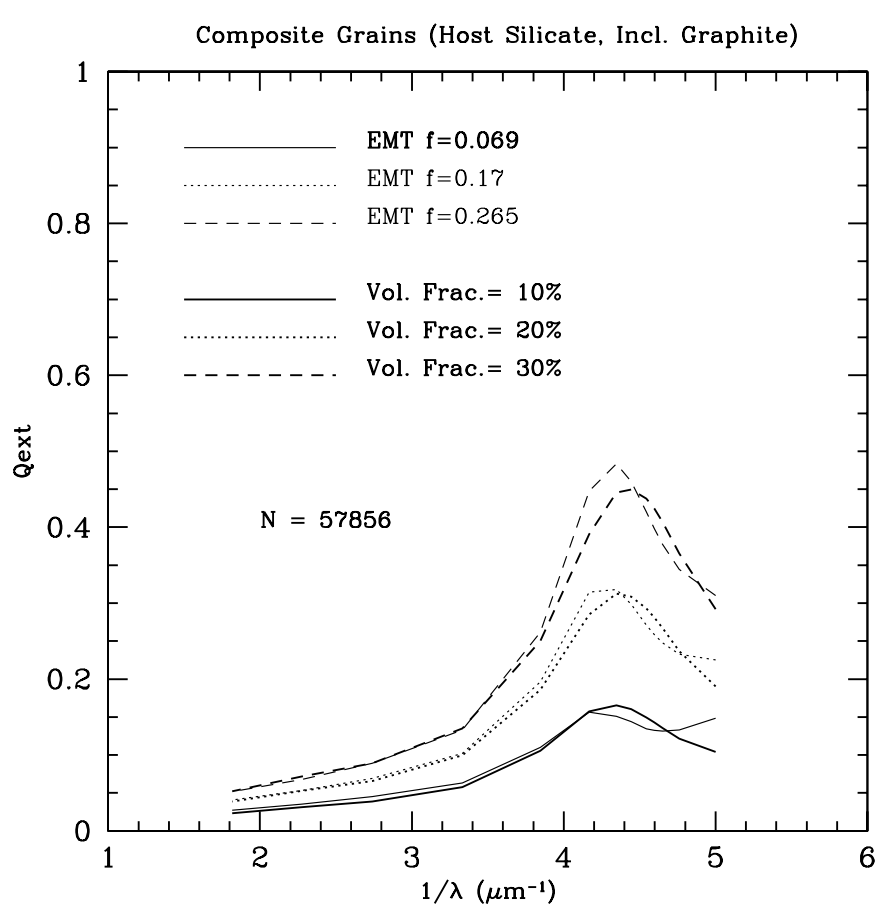

Fig. 3. Extinction efficiencies for the composite grain using the EMT approximation and DDA for three volume fractions.

It is seen that the wavelength of the peak extinction shifts towards longer wavelength as the volume fraction of inclusions is decreased. Figure 3d highlights this effect clearly for 57856 dipoles. The wavelength of peak extinction for the $30 \%$ volume fraction of inclusions shifts from about $0.22 \mu \mathrm{m}$ to $0.23 \mu \mathrm{m}$ for the $10 \%$ volume fraction of inclusions. These curves also show the variation in the width of the feature as a function of the volume fraction. In our previous study on the porous grain model, we also found the shift in the extinction peak towards longer wavelength as well as the variation in the width of the peak as the porosity was increased (Papers I and II). All these results indicate that the inhomogeneities within the grains (i.e. distribution of materials, porosity) play a very important role in producing the $2175 \AA$ feature.

Using the Maxwell-Garnet effective medium approximation (Bohren \& Huffman 1983) we have obtained the optical constants for the composite grains and have used these constants in Mie theory to calculate the extinction efficiencies of the composite grains. Figure 3 shows the extinction curves for the composite grain obtained using the EMT approximation along with the curves obtained using the DDA. It should be pointed out here that the fraction of the inclusions of graphite in the EMT approximation is obtained by the $\chi^{2}$ minimization of the bestmatched DDA curve and corresponding EMT curve. It is seen that EMT curves deviate from DDA curves near the peak and as the volume fraction increases this deviation also increases. However, the EMT results seem to be in agreement with the DDA results in the optical region $(0.55 \mu \mathrm{m}-0.30 \mu \mathrm{m})$. Voshchinnikov \& Mathis (1999) have found that their results on the composite grains obtained using the multilayered sphere EMT do not agree with the DDA results. The EMT results do not agree with the DDA results because the EMT does not take into account the inhomogeneities within the grain (e.g. internal structure, surface roughness, voids) (Wolff et al. 1994, 1998). Since Maxwell-Garnett mixing rules provide the extreme cases of the possible values of the effective dielectric constants of a two-component mixture (Chylek et al. 1999), we have used the Maxwell-Garnett method. However, it would still be very useful and advantageous to compare the DDA results for the composite grains (with varying inclusion sizes and volume fractions) with those computed by other EMT/Mie type series solution techniques in order to examine the applicability of several mixing rules (e.g. Chylek \& Srivastava 1983; Chylek et al. 1999; Voshchinikov \& Mathis 1999; Wolff et al. 1998).

We have also calculated the extinction efficiency for the composite grains for several inclusion sizes, " $n$ ", at a constant volume fraction but we did not find any shift in the extinction peak with the inclusion size.

Figure 4 shows extinction curves for four sizes of the composite grains; i.e. (a) $0.01 \mu$ (b) $0.05 \mu$ (c) $0.1 \mu$ and (d) $0.2 \mu$, in the spectral range $0.55 \mu \mathrm{m}-0.20 \mu \mathrm{m}$ for 3 models. It is seen from these curves that the extinction peak shifts as the grain size increases.

In addition to the composite grains, consisting of silicate and graphite, a component of small graphite grains is also required to produce the observed peak at $2175 \AA$ in the interstellar extinction curve. For this purpose we use our earlier results on porous graphite grains (see Fig. 3, Paper I)

\section{Composite grains and interstellar extinction}

We use the extinction efficiencies of the composite grains (made up of the host silicate and graphite inclusions) and small porous (about $20 \%$ porosity) graphite grains for a power law size distribution (i.e. $n(a) \sim a^{-3.5}$, Mathis et al. 1977) to evaluate the interstellar extinction curve in the wavelength region of $0.55-0.20 \mu$. For this calculation we have used two size distributions viz. (i) $a=0.001-0.100 \mu$ for grain models with $N=912$ and 7208 at three volume fractions of inclusions viz. $10 \%, 20 \%$ and $30 \%$ (ii) $a=0.005-0.250 \mu$ for grain models with $N=57856$ at two volume fractions of inclusions viz. $10 \%$ and $20 \%$. As mentioned earlier in order to reproduce the observed extinction peak at $2175 \AA$ we have included small porous (about 20\% porosity) graphite grains.

The average observed interstellar extinction curve (Savage \& Mathis 1979) is then compared with the model curve formed from a $\chi^{2}$ minimized and best fit linear combination of the composite and porous graphite grains in the following way:

The two (model) interstellar extinction curves for the composite and porous graphite grains are linearly combined as $p$ times (contributory fraction of composite grains) composite and $q$ times (contributory fraction of graphite grains) graphite to render a net curve for 


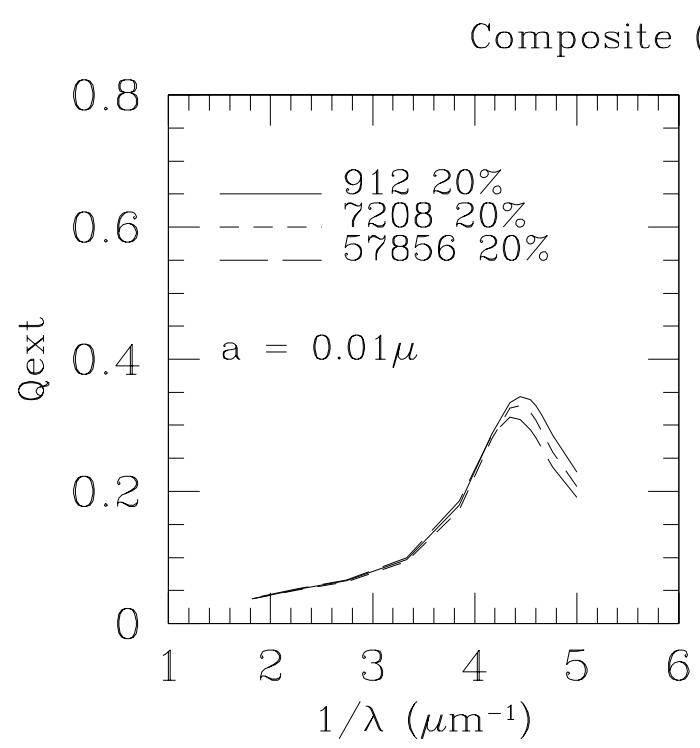

(Silicate \& Graphite)
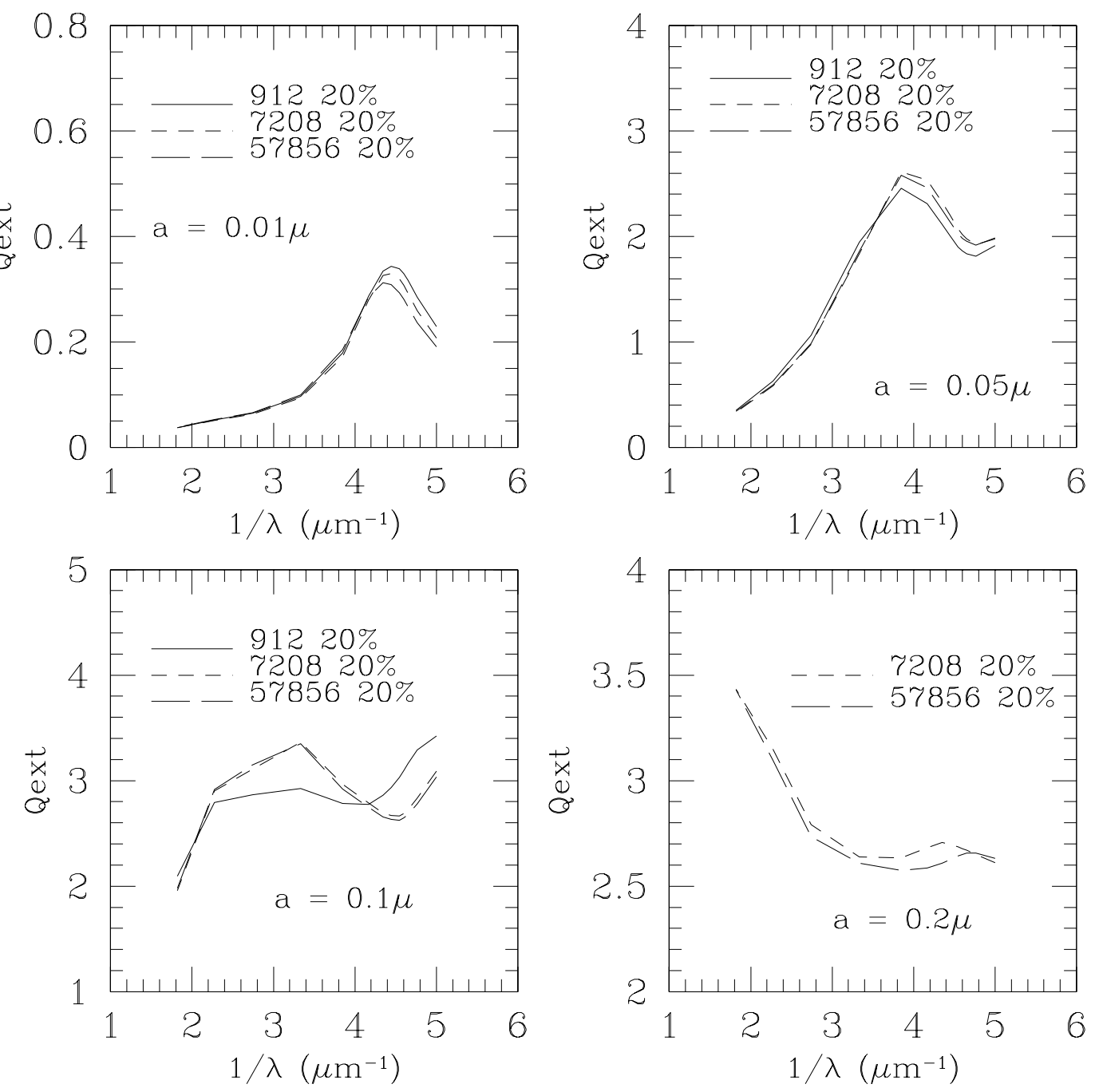

Fig. 4. Extinction efficiencies for the composite grains for four host sphere sizes: a) $0.01 \mu$, b) $0.05 \mu$, c) $0.1 \mu$ and d) $0.2 \mu$.

comparison with the observed curve (for details on the $\chi^{2}$ minimization method see Papers I and II).

The set of reduced $\tilde{\chi^{2}}{ }_{j}$ values is defined as (Bevington 1969):

${\tilde{\chi^{2}}}_{j}=\frac{\sum_{i=1}^{n}\left(S_{i}^{j}-T_{i}^{k}\right)^{2}}{p p}$

where $p p$ is the degrees of freedom, $S_{i}^{j}\left(\lambda_{i}\right)$ is the $j$ th model curve for the corresponding $p$ and $q$ linear combination of composite and graphite porous grains and $T_{i}^{k}\left(\lambda_{i}\right)$ is for the observed curve, $\lambda_{i}$ are the wavelength points with $i=1, n$ where $n$ are the number of wavelength points of the extinction curves.

The combination of $p$ and $q$ gives minimized $\chi^{2}$ values which are shown in Table 2.

Figures 5, 6, 7 show the interstellar extinction curves for the composite grains with $N=912,7208$ and 57856 respectively in the wavelength region of $0.55 \mu \mathrm{m}-0.20 \mu \mathrm{m}$. It is to be noted here that for the grain models with $N=912$ and 7208 we have considered small sizes
Table 2. Best fit parameters for the interstellar extinction curves for the composite dust models for 3 vol. fractions; $10 \%$, $20 \%, 30 \%$.

\begin{tabular}{lccc}
\hline \hline Parameters & $N=912$ & $N=7208$ & $N=57856$ \\
\hline$p(10 \%)$ & 0.2 & 0.2 & 0.3 \\
$q$ & 0.4 & 0.4 & 0.5 \\
$\chi^{2}$ & 0.0745 & 0.0823 & 0.1483 \\
\hline$p(20 \%)$ & 0.1 & 0.1 & 0.1 \\
$q$ & 0.5 & 0.5 & 0.6 \\
$\chi^{2}$ & 0.0913 & 0.0943 & 0.1936 \\
\hline$p(30 \%)$ & 0.1 & 0.1 & \\
$q$ & 0.5 & 0.5 & \\
$\chi^{2}$ & 0.1019 & 0.1031 & \\
\hline
\end{tabular}

$(0.001 \mu-0.100 \mu)$ for the composite grains that satisfy the DDA validity criteria. As a result, the models with $N=912$ and 7208 do not fit well in the optical region; i.e. $0.55 \mu \mathrm{m}-0.30 \mu \mathrm{m}$ but they fit well in the bump region; i.e. $0.23 \mu \mathrm{m}-0.20 \mu \mathrm{m}$. On the other hand, grain models with $N=57856$ and a size distribution of larger grains 


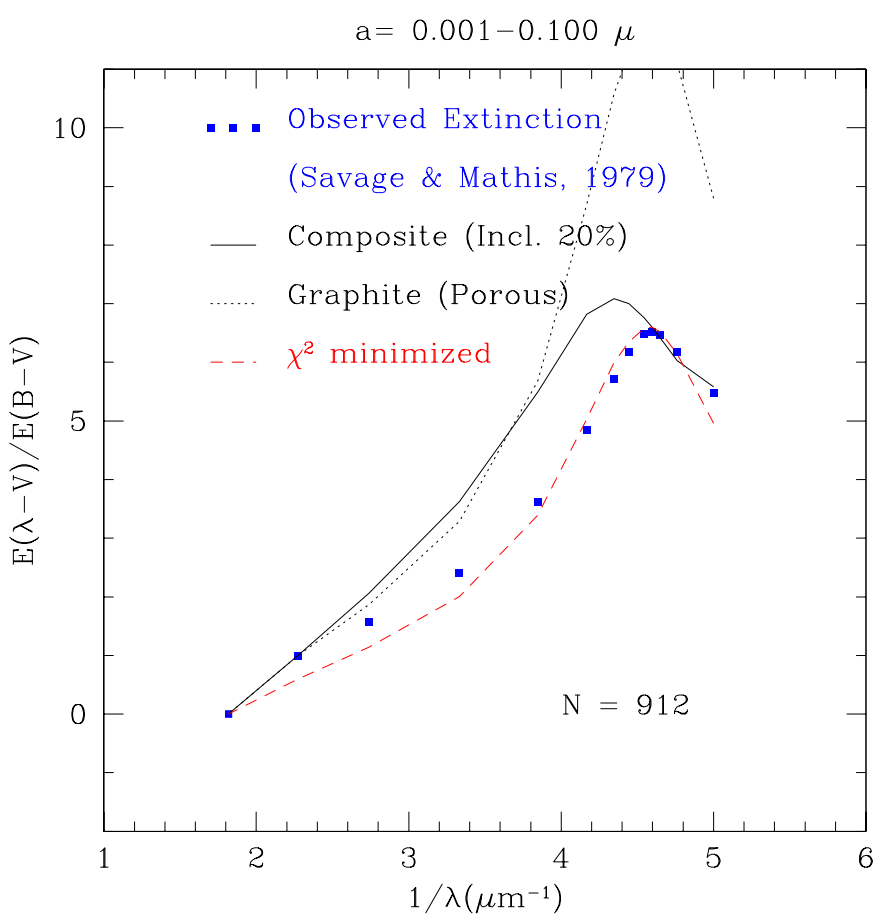

Fig. 5. Comparison of the observed interstellar extinction curve with the best fit model curve of composite grains $(N=$ 912 ) with $20 \%$ of volume fraction of inclusions and porous graphite grains.

(i.e. $0.005-0.250 \mu$ ) are found to fit better with the observed curve in the optical region but they do not fit well in the bump region. The best fit $\chi^{2}$ value in the optical region for the grain model with $N=57856$ is about 0.07 ; which is as good as that found for the other two models. At present, we have restricted the calculations in the wavelength region of $0.55 \mu \mathrm{m}-0.20 \mu \mathrm{m}$ with more emphasis on the bump region. We plan to study the extinction properties for the composite grains with $N \gtrsim 50000$ and a grain size distribution of $0.001 \mu-0.250 \mu$ in the wavelength region $3.4 \mu \mathrm{m}-0.10 \mu \mathrm{m}$. The results (Figs. 5, 6, 7 and Table 2) on the composite grains also indicate that about $80 \%-90 \%$ silicate accounts for the observed extinction; the amount of graphite required is very small.

Mathis (1996) and Dwek (1997) have proposed composite fluffy dust models (CFD) to overcome the cosmic abundance constraints. Since the fluffy aggregates can produce more extinction per unit mass than their combined individual constituent dust grains, an interstellar dust model with fluffy aggregates as a major dust component will require a minimal amount of carbon and silicate dust. Using the EMT, Mathis (1996) has studied the optical properties of the composite dust consisting silicates, amorphous carbon and graphite. Combining these composite grains and a separate component of graphite grains, he has obtained the cosmic carbon abundance, $\mathrm{C}$ atoms per $10^{6} \mathrm{H}$ atoms, $\mathrm{C} / \mathrm{H}$, of about $140-160$; considerably lower than that predicted by the solid grain model, $\gtrsim 300$ ppm (Mathis et al. 1977) but it is still significantly above the value of 110 implied from the recent ISM val-

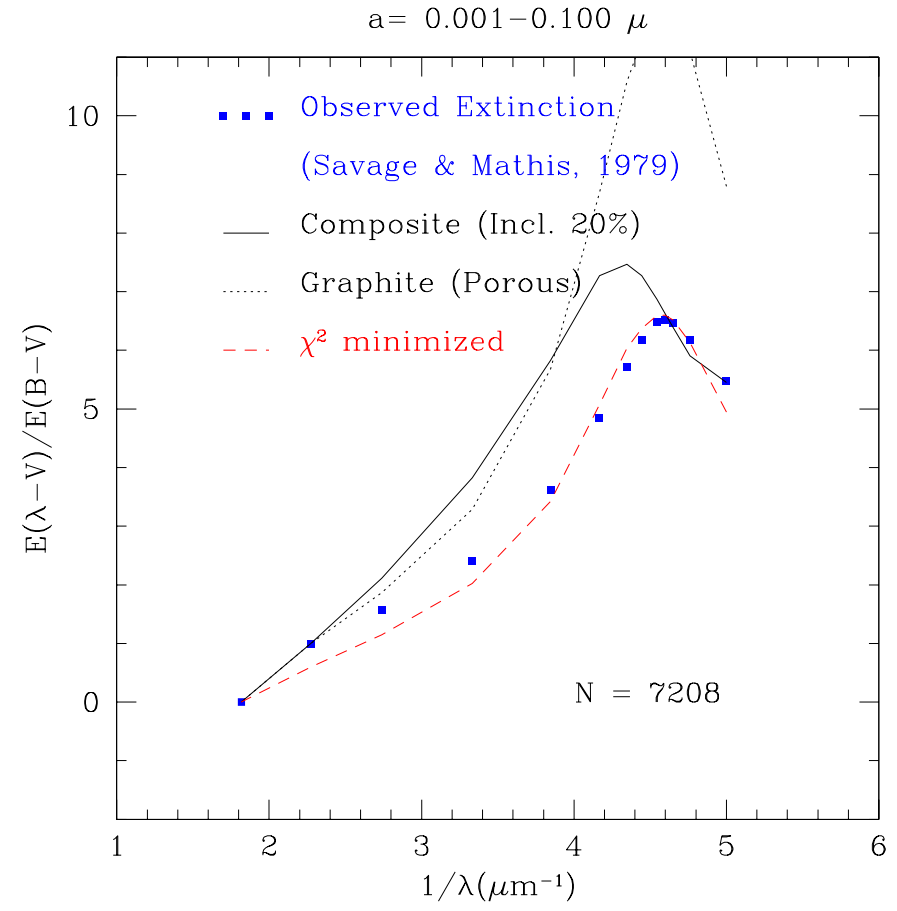

Fig. 6. Comparison of the observed interstellar extinction curve with the best fit model curve of composite grains $(N=$ 7208 ) with $20 \%$ of volume fraction of inclusions and porous graphite grains.

ues (Mathis 2000). From the composite grain models we have proposed here, we estimate $\mathrm{C}$ abundance to be between 150-170. In a recent review, Snow (2000) has addressed the issues related to and the question of appropriate reference abundance standards and has noted that no model for the dust extinction copes successfully with the reduced quantities of available elements imposed by the revised cosmic abundance standards and the consequent reductions in depletions.

\section{Summary and conclusions}

The composite dust models, made up of the host silicate spheres and graphite inclusions, are generated using the modified DDA code (Dobbie 1999). The DDA validity criteria for each model; i.e. with $N=912,7208$ and 57856, are presented. The optical properties for the composite grains are studied in the wavelength region of $0.20 \mu \mathrm{m}-0.55 \mu \mathrm{m}$. In particular, the extinction is studied as a function of the inclusion size and volume fraction. Our results show that the wavelength of peak extinction shifts towards longer wavelength as the volume fraction of the inclusion decreases. These results indicate that the inhomogeneity within the grains can produce the shift in the central wavelength of the extinction bump as well as variation in the width of the bump. The wavelength of peak extinction does not seem to shift with the size of the inclusion. Using the extinction efficiencies of the composite grain models and the graphite grains we have evaluated the interstellar extinction curves in the wavelength region of $0.55 \mu \mathrm{m}-0.20 \mu \mathrm{m}$. The model curves 


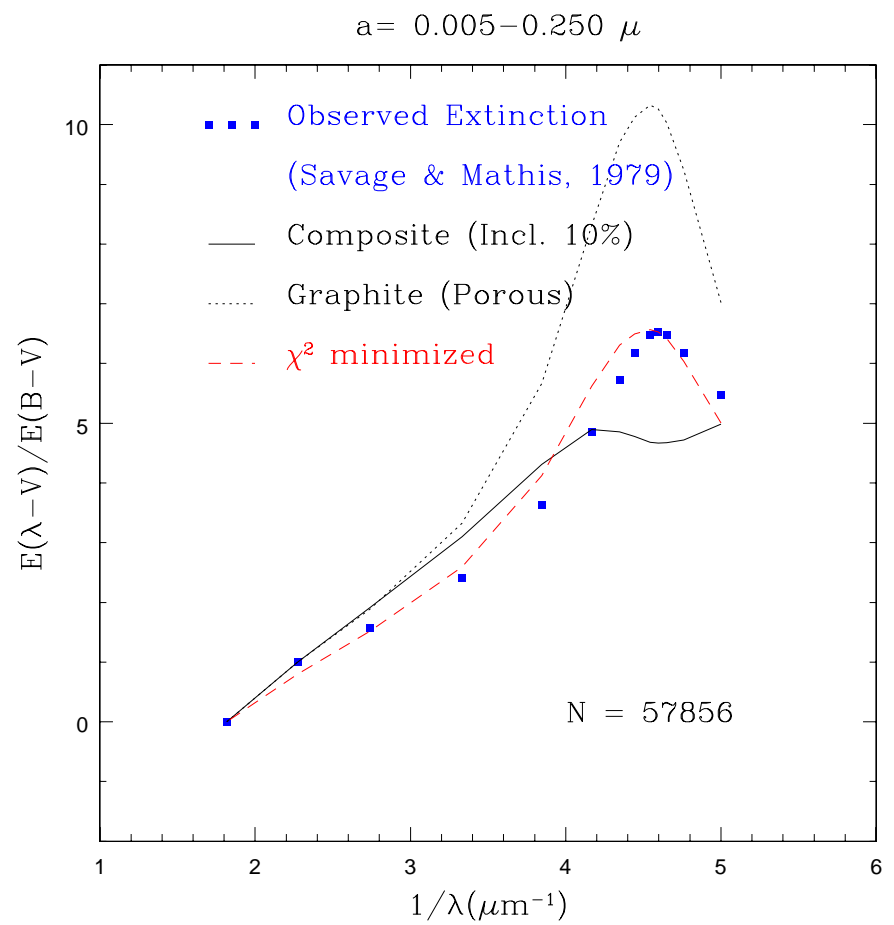

Fig. 7. Comparison of the observed interstellar extinction curve with the best fit model curve of composite grains $(N=$ 57856 ) with $10 \%$ of volume fraction of inclusions and porous graphite grains.

are found to fit the observed extinction reasonably well. We plan to extend these calculations on the composite grains with $N \gtrsim 50000$ and the grain size distribution of $0.001 \mu-0.250 \mu$ to reproduce the observed extinction in the entire spectral region of $3.4 \mu \mathrm{m}-0.10 \mu \mathrm{m}$. However, these DDA calculations would require considerable computer memory and cpu time. It would be, therefore, very useful to develop the effective medium theory with Mietype series solutions for the composite grains (e.g. Wolff et al. 1998, Voshchinikov \& Mathis 1999). We also plan to study the scattering properties (viz. scattering phase function, polarization, asymmetry parameter, albedo) for the composite grains and use these results to interpret the observed scattering from the cometary dust.

Acknowledgements. The authors thank the referee for very useful and constructive comments on the paper. We thank Bruce Draine and Piotr Flatau for providing the DDA code. D. B. V. thanks Dalhousie University for the support and hospitality during his visits to Halifax, Canada. The present study on composite grains was initiated at Dalhousie University.

\section{References}

Bevington, P. R. 1969, Data Reduction and Error Analysis for the Physical Sciences (New York: McGraw-Hill), Chapter $1-4$

Bohren, C. F., \& Huffman, D. R. 1983, in Absorption and Scattering of Light by Small Particles (John Wiley, NewYork)

Chylek, P., \& Srivastava, V. 1983, Phys. Rev. B, 27, 5098

Chylek, P., Videen, G., Geldart, D. J. W., Dobbie, J., \& William Tso, H. C. 1999, in Light Scattering by Nonspherical Particles, ed. M. Mishchenko, J. W. Hovenier, \& Travis L. D. (Academic Press, USA), 274

Defoe, H. 1965, J. Chem. Phys., 43, 3199

Dobbie, J., \& Chylek, P. 1998, in Preprints of Conf. on Light Scattering by Small Particles, ed. M. Mishchenko, J. W. Hovenier, \& L. D. Travis, 155

Dobbie, J. 1999, Ph.D. Thesis, Dalhousie University

Draine, B. T. 1985, ApJS, 57, 587

Draine, B. T. 1987, Preprint Princeton Observatory, No. 213

Draine, B. T. 1988, ApJ, 333, 848

Draine, B. T., \& Flatau, P. J. 1994, Opt. Soc. Am., 11, 1491

Draine, B. T., \& Flatau, P. J. 1998, DDA code version ddscat5a9

Dwek, E. 1997, ApJ, 484, 779

Mathis, J. S., Rumpl, W., \& Nordsieck, K. H. 1977, ApJ, 217, 425

Mathis, J. S. 1996, ApJ, 472, 643

Mathis, J. S. 2000, JGR, 105, 10269

Mathis, J. S., \& Whiffen, G. 1989, ApJ, 341, 808

Perrin, J. M., \& Lamy, P. L. 1990, ApJ, 364, 146

Purcell, E. M., \& Pennypacker, C. R. 1973, ApJ, 186, 705

Savage, B. D., \& Mathis, J. S. 1979, Ann. Rev. A\&A, 17, 73

Shapiro, P. R. 1975, ApJ, 201, 151

Snow, T. P., JGR, 105, 10239

Vaidya, D. B., \& Gupta, R. 1997, A\&A, 328, 634 (Paper I)

Vaidya, D. B., \& Gupta, R. 1999, A\&A, 348, 594 (Paper II)

Vaidya, D. B., Gupta, R., Dobbie, J. S., \& Chylek, P. 2000, in Preprints of Conference on Light Scattering by Nonspherical Particles, ed. G. Videen, Q. Fu, \& P. Chylek, 253

Voshchinnikov, N. V., \& Mathis, J. S. 1999, ApJ, 526, 257

Wolff, M. J., Clayton, G. C., Martin, P. G., \& Sculte-Ladlback, R. E. 1994, ApJ, 423, 412

Wolff, M. J., Clayton, G. C., \& Gibson, S. J. 1998, ApJ, 503, 815

Yung, Y. L. 1978, Appl. Opt., 17, 3707 